they perform a regulatory role in the cell or is their synthesis merely a metabolic safety valve? The most compelling evidence that they are involved in regulation comes not from the stringent response-which may be regarded simply as an emergency shutdown of many cellular functions-but from the observation of Lazzarini et al. (J. Biol. Chem., 246, 4381; 1971) that the concentration of ppGpp in cells growing normally varies inversely with the growth rate, and coincidentally with the rate of stable RNA accumulation.

The crucial problem thus centres on the nature of the target of ppGpp in its regulatory role in the context of RNA synthesis. Apart from various enzymes involved in nucleotide metabolism ppGpp is known to interact with and inhibit one component of the transcription machinery, the RNA polymerase itself, and also two components of the protein synthesis machinery, the initiation factor IF2, and the elongation factor $\mathrm{Tu}$. In the latter cases ppGpp seems to act quantitatively as an analogue of GDP whereas in the former case ppGpp is a far stronger inhibitor than GDP.

Does the interaction of ppGpp with any of these proteins directly regulate the synthesis of stable RNA? Haseltine (Nature, 285, 329; 1972) has shown that the proportion of rRNA synthesized by highly purified RNA polymerase in vitro is not significantly affected by ppGpp. The effect, if any, of IF 2 on the specificity of transcription is unknown. The elegant equation of the putative rRNA synthesis control factor, psi, with the elongation factor Ts Tu (Blumenthal, Landers and Weber, Proc. US Nat. Acad. Sci., 69,1313 ; 1972) points, however, to a mode of positive control of stable RNA synthesis mediated by a component of the protein synthesis machinery. The activity of the control element would be negatively regulated by the concentration of ppGpp relative to that of GTP. If Tu should be confirmed as a regulatory target of $\mathrm{ppGpp}$-and a sine qua non of confirmation is minimally a demonstrable functional association of RNA polymerase and Tu -a molecular mechanism for the coupling of stable RNA production to protein synthesis would have been formulated from the concerted labours of the past twenty years.-A. A. T.

\section{DEMOGRAPHY}

\section{Change in Ireland}

THE past decade has seen a rapid reduction in the Irish Republic in the age of marriage which, according to Brendan M. Walsh (Demography, 9, 187 ; 1972), means that Jreland "will before long cease to be remarkable by European standards in regard to nuptiality". For most of the past century, Ireland has been remarkable not merely for its late age of marriage but also for the proportions of men and women remaining unmarried, in which circumstances it is surprising that Walsh says that the only reliable information on age of marriage in Ireland before 1957 was a special inquiry into marriages of less than one year's duration included in the 1946 census. That inquiry showed that in the Republic of Ireland as a whole, the median age of marriage was 27.0 years for women and 31.8 years for men (with remarriages included). By 1957, the median age at first marriage was 25.9 for women and 29.4 for men, representing a small decrease since 1946 when remarriages were included.

In the remainder of the $1950 \mathrm{~s}$, the median ages of marriage remained the same, but in the 1960 s there seems to have been a quite rapid decrease of median marriage age, which amounted in 1969 to 23.8 years for women and 26.0 years for men. Walsh points out that the contrast between the 1950 s and the 1960 s corresponds with the difference in the pace of economic growth of the two periods, which amounted to 30 per cent (in real terms) per head between 1946 and 1957 and to 46 per cent between 1957 and 1968. During the whole period, the reduction of the age of first marriage of men has been greater than that of women, with the result that the difference between the median ages of men and women at first marriage has decreased from 4.8 years in 1946 to 2.2 years in 1967.

The well known difference between the marriage patterns in towns and in the countryside persists, but not all the change in the pattern of nuptiality in Ireland since 1946 can be accounted for by the displacement of farming people to the towns. In 1946, the median age of marriage for women was 25.8 years in the towns and 28.0 years in the country (the corresponding figures for men are 29.2 years in towns and 33.6 in rural areas). From Walsh's analysis of marriage patterns in terms of occupation, it is apparent that even among the farmers, who are traditionally the last to marry, the median age of marriage has decreased from 36.1 years in 1946 to 31.7 years in 1969. Among farm labourers, the median age at marriage had decreased to 27.4 years, substantially the same as the median age at marriage of professional men in Ireland. Farm labourers remain conspicuous, however, because of the high proportion remaining unmarried-in 1966, sixty per cent of farm labourers over the age of 14 were unmarried.

Walsh points out that the frequency distribution of age at marriage is highly skewed, especially for women. Over the years, however, there has been a tendency for the age at marriage to conform more closely with the mode-in other words, smaller proportions of men

\title{
Revealing C-type Viruses
}

ACCORDING to the doctrine of Huebner and Todaro all cells inherit genes capable of inducing a transformation to malignancy, so-called oncogenes, and also genes capable of specifying a Ctype virus, so-called virogenes. When both oncogenes and virogenes are expressed the result is a malignant cell releasing RNA tumour viruses, whereas when oncogenes alone are expressed the cell is transformed but fails to release virus. Any cell infected and transformed by an exogenous RNA tumour virus should, according to this hypothesis, have two sets of oncogenes and virogenes. Experiments which can be interpreted in this way are reported in the next issue of Nature New Biology (August 23) by Klement and six of his colleagues.

Klement et al. exposed a clone of rat cells of the XC cell line to 5-bromodeoxyuridine (BrUdR) and as a result induced the production of an RNA C-type virus which contains reverse transcriptase. During its ten-year history the XC cell line, originally isolated by Svoboda, who raised a tumour in a newborn Wistar rat with the Prague strain of Rous (avian) sarcoma virus, has been repeatedly passaged in vivo and in vitro. $\mathrm{XC}$ cells contain the group specific antigen of the avian tumour viruses, and the Rous genome can be rescued by fusing these rat cells with chicken cells. On their own the XC cells do not produce Rous virus.

Several tests show, however, that the virus induced by Klement et al. from their clone with BrUdR is not the Prague Rous sarcoma virus. Rather it seems to be a rat virus, for it crossreacts with antisera to the rat species specific gs-1 antigen; the virus does not, however, transform or cause any detectable cytopathic effect in the rat cells that have so far been tested. These $\mathrm{XC}$ cells, as Klement et al. suggest from these findings, may well contain two sets of genes capable of specifying a C-type RNA virus, exogenous avian and endogenous rat. 